\title{
J. Reaven, A. Bleakes-Smith, S. Nichols and S. Hepburn: Facing Your Fears: Group Therapy for Managing Anxiety in Children with High-Functioning Autism Spectrum Disorders (Facilitator Manual and Parent/Child Workbooks)
}

\author{
Paul H. Brookes, Baltimore, 2011, xiii, 169 pp. ISBN 978-1598571783 (paper)
}

\section{Eli R. Lebowitz}

Published online: 4 September 2011

(C) Springer Science+Business Media, LLC 2011 education, cognitive restructuring, relaxations training and the creation and completion of a hierarchy of behavioral exposures to previously avoided situations. The novelty of the contribution is in the painstaking efforts made by the authors to present all these in formats that recognize the special challenges of children with autism. For example, while identifying calming behaviors that can be useful in stressful situations is common to many anxiety treatments, the authors are careful to recognize the natural tendency of developmentally challenged children to have a very constrained repertoire of such soothing behaviors and they direct the therapist to identify diverse behaviors that can be appropriate for a variety of settings. The consistently clear and precise language, the usage of many visual cues in the child's workbook and the practice of preceding tasks with clear examples that a child can mimic to be successful also exhibit the author's experience in creating content that will be easily adopted by children with autism.

The choice to focus on group rather than individual treatment is a daring one when targeting children with autism. The benefits, such as presenting the children with opportunities for social skills learning and creating an ad hoc support group for additional non-anxiety related difficulties, possibly balanced by the need to overcome significant social challenges in order to address the anxiety disorder. Future research will hopefully address the issue but there appears not to be any significant barrier to implementing the program in an individual setting if necessary.

Another remaining question is the suitability or adaptability of the program for children with lower functioning autism. As many of these children also experience significant anxiety it is my hope that the authors will turn their attention to them as well and continue their work of bringing children with autism within the fold of those who may profit from the advances we have made in CBT for pediatric anxiety. 